\title{
A logic for epistemic two-dimensional semantics*
}

\author{
Peter Fritz \\ Final Draft
}

\begin{abstract}
Epistemic two-dimensional semantics is a theory in the philosophy of language that provides an account of meaning which is sensitive to the distinction between necessity and apriority. While this theory is usually presented in an informal manner, I take some steps in formalizing it in this paper. To do so, I define a semantics for a propositional modal logic with operators for the modalities of necessity, actuality, and apriority that captures the relevant ideas of epistemic two-dimensional semantics. I also describe some properties of the logic that are interesting from a philosophical perspective, and apply it to the so-called nesting problem.
\end{abstract}

\section{Epistemic Two-Dimensional Semantics}

The term "two-dimensional semantics" has been applied to a variety of theories, an overview of which can be found in Schroeter (2010). E.g., a popular way of specifying the semantics of indexical expressions uses intensions that are relativized to two indices, and is therefore sometimes called a two-dimensional semantics. Such a semantics is discussed, e.g., in Kamp (1971) and Kaplan (1989). Another example is the pragmatic theory proposed in Stalnaker (1978), which evaluates sentences relative to two indices to give a formal account of assertion. In this paper, I will be concerned with the variant proposed by David Chalmers, which he calls epistemic two-dimensional semantics, e.g., in Chalmers (2004). It can be seen as a framework dealing with meaning and its connection to necessity and apriority, as well as to issues of reference determination and context dependence. Chalmers has also used it in an argument concerning the problem of consciousness, in Chalmers (1996), and in a discussion of propositional attitude ascriptions, in Chalmers (2011b). Unless indicated otherwise, I will only be talking about epistemic two-dimensional semantics from now on, and therefore drop the qualifier epistemic.

In this section, I introduce the relevant ideas of two-dimensional semantics and present the nesting problem. In section 2 , I formally define the logic for two-dimensional semantics with which this paper is concerned. I discuss some philosophically interesting properties and possible variants of this logic in section 3. Section 4 contains a discussion of the nesting problem, and section 5 provides a concluding summary.

\footnotetext{
*Forthcoming in Synthese. The final publication is available at http://link.springer.com/article/10.1007\%2Fs11229-013-0260-x.
} 
I start by introducing two-dimensional semantics. Central to two-dimensional semantics is the connection between meaning and the modalities of necessity and apriority. Concerning these two notions, Chalmers simply notes that possible (not necessarily not the case) is what might have been the case (Chalmers, 2004 , p. 210), and explains that roughly, a priori is what "can be conclusively non-experientially justified on ideal rational reflection" (Chalmers, 2004, p. 208).

In standard intensional semantics, the meaning of an expression is modeled by a function, called its intension, that maps possible worlds to extensions. Taking the extensions of sentences to be truth values, necessity is then analyzed as having an intension that maps every world to the truth value true. One of the central claims of two-dimensional semantics is that both necessity and apriority can be analyzed in such a manner. To do so, two-dimensional semantics postulates two kinds of possibilities. The first are the familiar (possible) worlds, which can be understood as ways the world could be. The second are called scenarios, and can be understood as ways the world might be, given what can be known a priori. It is also assumed that every scenario is associated with a unique corresponding world, where two scenarios might be associated with the same world. The evaluation of expressions is now relativized to both worlds and scenarios, and it is this kind of double-indexing from which the theory takes its name. More precisely, two-dimensional semantics models the meaning of an expression by a function that maps every pair containing a world and a scenario to an extension. I will call this function the two-dimensional intension.

We can visualize two-dimensional intensions as tables or matrices, where worlds are listed on the horizontal axis, scenarios are listed on the vertical axis, and the appropriate extensions are written in the cells. This is why twodimensional intensions are also called two-dimensional matrices. Let me illustrate this with the sentence "Hesperus is Phosphorus", assuming that Kripke (1980 [1972]) is correct in claiming that this is necessary and a posteriori. Let $s$ and $t$ be scenarios, and $w$ and $v$ their associated worlds, $s$ being the actualized scenario and $w$ the actual world. Then ignoring the other scenarios and worlds, the two-dimensional intension of "Hesperus is Phosphorus" might look as in Table 1:

\begin{tabular}{l|ll} 
& $w$ & $v$ \\
\hline$s$ & true & true \\
$t$ & false & false
\end{tabular}

Table 1: "Hesperus is Phosphorus"

When visualizing two-dimensional intensions in this way, we can describe the analysis of necessity as requiring the table to contain true throughout the horizontal at the actualized scenario. Similarly, we can visualize the analysis of apriority as requiring the table to contain true throughout the diagonal, although this has to be refined if there are multiple scenarios associated with the same world. In the particular example of "Hesperus is Phosphorus" given above, all of the cells in the horizontal at the actualized scenario $s$ contain true, so "Hesperus is Phosphorus" comes out as necessary. Since the diagonal contains a false, it also comes out as not being a priori.

To state these analyses of necessity and apriority without appeal to tables, we can derive two (one-dimensional) intensions from a two-dimensional intension: 
The primary intension is defined as the function that maps every scenario to what the pair containing it and its possible world is mapped to by the twodimensional function. The secondary intension is defined as the function that maps every world $w$ to what the pair containing the actualized scenario (the scenario that is in fact realized) and $w$ is mapped to by the two-dimensional function. Apriority is then analyzed as having a constant primary intension mapping every scenario to true, and necessity is analyzed as having a constant secondary intension mapping every world to true.

What I have said so far is of course only an outline of two-dimensional semantics, but it will suffice for present purposes. Two-dimensional semantics has been criticized on a number of grounds. Some authors have argued in various ways that two-dimensional semantics "generates difficulties for a systematic combinatorial semantics" (Schroeter, 2010, section 2.4.2). In particular, this line of argument has been pursued by Scott Soames, who has given a series of arguments which are supposed to exhibit problems of this kind for twodimensional semantics. One of these arguments, described in (Soames, 2005, see Argument 5 on pp. 278-279), was discussed in a more abstract form in (Dever, 2007, see Version 2 on p. 11), and has been reformulated in its most perspicuous form in (Chalmers, 2011b, endnote 25). In Chalmers's rendition, it is the following schematic argument, consisting of two premises, (A1) and (A2), and a conclusion, (A3):

(A1) If it is a priori that $p$, then it is necessarily a priori that $p$.

(A2) Necessarily, if it is a priori that $p$, then $p$.

(A3) If it is a priori that $p$, then it is necessary that $p$.

The conclusion follows from the premises by standard modal reasoning: As (A2) is a necessitated implication, we can infer that the necessitated antecedent implies the necessitated consequent, from which (A3) follows with (A1) by chaining of implications. According to Soames's discussion of his version, twodimensional semantics entails the truth of all instances of the first premise. Proponents of two-dimensional semantics usually assume that the conclusion has false instances; in fact, this is one of the motivations for developing twodimensional semantics. Soames also assumes that all instances of the second premise are true, and therefore takes the argument to be a refutation of twodimensional semantics (Soames, 2005, p. 325). Dever does not draw such a strong conclusion, but notes that the problem raises the "important and imposing challenge" of giving "a detailed story about how [primary and secondary intensions] compose and interact" (Dever, 2007, p. 16). I agree with Dever in this, and I will give such a story in the form of a semantics for a modal logic in the next section. Since the crucial element of the above argument is the nesting of "a priori" inside the scope of "necessarily", I follow Dever in calling it the nesting argument, and Chalmers in calling the problem it poses for twodimensional semantics the nesting problem. (Closely related arguments have also been discussed in (Glüer and Pagin, 2006, section 5), Forbes (2011), Glüer and Pagin (2012) and Michels (2012). In the following, I will mostly confine myself to the nesting argument as described above.) 


\section{Formal Syntax and Semantics}

The alleged difficulties in providing a systematic semantics as well as the more specific nesting problem motivate spelling out the details of two-dimensional semantics in a rigorous form. By giving a formal system that captures the central claims of two-dimensional semantics, we can answer Dever's challenge and provide the desired detailed story about how the different intensions interact. If this system provides an explicit treatment of the modalities of necessity and apriority as well as the usual Boolean connectives, we can also represent the nesting problem in it, which might shed some light on how to solve it. Finally, it is interesting just to see how the modalities of necessity and apriority relate and interact according to two-dimensional semantics. This might also provide a way of testing two-dimensional semantics, by checking whether these relations and interactions are in accord with our understanding of necessity and apriority. The most natural way of developing such a formal system is in the form of a modal logic.

One might wonder whether such a logic has not already been defined. And in fact, for some of the uses just mentioned, this is the case. E.g., the logics in both Segerberg (1973) and Davies and Humberstone (1980) show that relativizing truth to two different indices does not prevent us from giving a formal semantics. But neither of them provides a formalization of two-dimensional semantics, or is even intended to represent the notion of apriority, which is of crucial importance here (see also (Humberstone, 2000, p. 278)). Similarly, Michels (2012) defines a formal system to analyze another argument by Soames against two-dimensional semantics. While this can be used to argue that some aspects of two-dimensional semantics can be systematized, his system does not include an operator for apriority either, and can therefore not be used to represent the nesting problem, nor to systematically investigate the relations between necessity and apriority.

The one text that presents a logic that fits the description given above is Restall (2012). Essentially, Restall presents the same logic as the one defined below, since apart from using different symbols, he uses the same syntax, and his semantics is easily seen to be equivalent to the one considered here. But the present paper differs from Restall's in substantial respects. The main difference is that here, the model theory is based on the ideas of two-dimensional semantics, and used to define a logic that is then applied to the nesting problem, whereas Restall starts with independently motivated rules of a kind of sequent calculus, which he then uses to define a model theory that is intended to motivate some aspects of two-dimensional semantics.

So let me now start to develop a formal logic with modal operators for necessity and apriority according to the ideas of two-dimensional semantics. Since quantification in modal contexts is a notoriously thorny issue, and one that is not essential for present purposes, I will consider a propositional logic. As the interaction of indexicals with necessity and apriority in two-dimensional semantics is also interesting, I am going to include a modal operator representing "actually" in the logic. Accordingly, I will consider a propositional modal language with operators for necessity, apriority and actuality. I use lower-case letters like $p$ and $q$ as proposition letters; $\neg$ and $\wedge$ as primitive Boolean operators; and $\square$ for necessity, $A$ for apriority and @ for actuality. As usual, other common Boolean operators are treated as syntactic abbreviations. Similarly, I use $\diamond$ for the dual of $\square(\neg \square \neg)$. Since to be possible is to have a negation which is not necessary, 
we can read $\diamond$ as possibly. Analogously, (Chalmers, 2004, p. 219) proposes that to be conceivable is to have a negation which is not a priori, which motivates reading the dual of $A(\neg A \neg)$ as conceivably. I therefore use the symbol $C$ for it, although I do not want to commit myself to the claim that apriority and conceivability are duals in this way, and that $C$ should therefore be read as conceivably.

To try to capture the ideas of two-dimensional semantics in a formal semantics, I will start with a class of Kripke frames. A Kripke frame for our language is a tuple $\left\langle W, R_{\square}, R_{@}, R_{A}\right\rangle$, where $W$ is a set and any $R_{\nabla}$ is a binary relation on $W$. (I use $\nabla$ as a variable ranging over $\square$, @ and A.) A Kripke model based on such a frame is obtained by adding a valuation function $V$, which is a function that maps every proposition letter to a subset of $W$. We call $W$ the set of points, and the relations $R_{\nabla}$ the accessibility relations. Note that points need not represent possible worlds, and in fact will not do so in the class of frames used below - they will represent tuples containing a world and a scenario. Truth of a formula $\varphi$ in a model $\mathfrak{M}=\left\langle W, R_{\square}, R_{@}, R_{A}, V\right\rangle$ is relativized to a point $w$, and written $\mathfrak{M}, w \Vdash \varphi$. This is defined recursively with the following clauses:

$$
\begin{aligned}
& \mathfrak{M}, w \Vdash p \text { iff } w \in V(p) \\
& \mathfrak{M}, w \Vdash \neg \varphi \text { iff not } \mathfrak{M}, w \Vdash \varphi \\
& \mathfrak{M}, w \Vdash \varphi \wedge \psi \text { iff } \mathfrak{M}, w \Vdash \varphi \text { and } \mathfrak{M}, w \Vdash \psi \\
& \mathfrak{M}, w \Vdash \nabla \varphi \text { iff } \mathfrak{M}, v \Vdash \varphi \text { for all } v \in W \text { such that } w R_{\nabla} v
\end{aligned}
$$

Modal logics can be characterized by classes of frames. For such a class $C$, we define a formula $\varphi$ to be valid on $C$, written $C \Vdash \varphi$, if it is true at any point in any model based on any frame in $\mathrm{C}$; and $\varphi$ to be a consequence of a set of formulas $\Gamma$ over $C$, written $\Gamma \vDash_{C} \varphi$, if $\varphi$ is true at any point in any model based on any frame in $C$ at which all elements of $\Gamma$ are true.

I will now define a class of frames based on the ideas of two-dimensional semantics and call them matrix frames. As two-dimensional intensions assign truth values to sentential expressions relative to world-scenario pairs, it would be natural to use frames in which the set of points is the Cartesian product $P \times S$ of two sets $P$ and $S$, one representing worlds and one representing scenarios. However, to make the formal semantics as simple as possible, I will use the same set to represent both worlds and scenarios, and therefore use frames in which the set of points is the Cartesian square $S \times S$ of some set $S$. This is a simplification, since it is plausible that there are in fact several scenarios that are associated with the same world. I will justify the use of these frames below by showing that this simplification makes no difference to the logic of the language considered here.

Thinking of the Cartesian square of a set as a table analogously to how twodimensional intensions were illustrated above, we want to interpret necessity as truth throughout the horizontal and apriority as truth throughout the diagonal. As the evaluation clause of modal operators in Kripke semantics universally quantifies over the points accessible from the point of evaluation, we therefore want every point to be be able to access every other point on the same horizontal via the relation interpreting the necessity operator, and every point to be able to access every point on the diagonal via the relation interpreting the apriority operator. How to define the accessibility relation for the actuality operator is less 
obvious. On the standard semantics for "actually" in which truth is relativized to a world of evaluation and a world of utterance, its semantic effect is to set the world of evaluation to the world of utterance; this is how it is formalized, e.g., in Kaplan (1989). According to two-dimensional semantics, we evaluate expressions relative to a scenario by assuming the scenario to be actual, which suggests that the semantic effect of "actually" is to evaluate the complement clause at the world of the current scenario. This means that the accessibility relation interpreting the actuality operator should relate every point to the point representing the scenario of the original point and the world of that scenario. In other words, we want every point to be be able to access just the point on the diagonal that is on the same horizontal.

To illustrate this, consider a frame containing nine points, the elements of the Cartesian square of a three-element set. Below, this is drawn as a table, and the points accessible from the upper middle point via the three relations are indicated using three kinds of arrows:

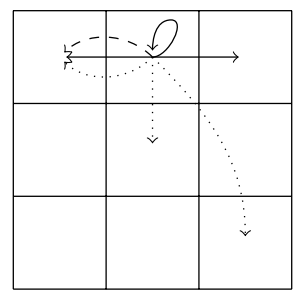

$$
\begin{aligned}
& \square: \longrightarrow \\
& \text { @: - -> } \\
& A: \cdots>
\end{aligned}
$$

Figure 1: Relations in a matrix frame

More formally, we give the following definition, making the arbitrary choice of taking the first element of a pair to represent the world, and the second the scenario:

Definition 1. A matrix frame is a frame $\mathfrak{F}=\left\langle W, R_{\square}, R_{@}, R_{A}\right\rangle$, where $W=$ $S \times S$ for some set $S$, and the relations are given by the following conditions:

- $\langle x, y\rangle R_{\square}\left\langle x^{\prime}, y^{\prime}\right\rangle$ iff $y=y^{\prime}$

- $\langle x, y\rangle R_{@}\left\langle x^{\prime}, y^{\prime}\right\rangle$ iff $y=y^{\prime}$ and $x^{\prime}=y^{\prime}$

- $\langle x, y\rangle R_{A}\left\langle x^{\prime}, y^{\prime}\right\rangle$ iff $x^{\prime}=y^{\prime}$

We say that $\mathfrak{F}$ is based on $S$. Let $\mathrm{M}$ be the class of matrix frames.

As noted before, formal models in which the evaluation of formulas is relativized to tuples or two indices are well known in the literature and have been studied in detail, e.g., in some of the works cited at the beginning of this section. What is new about the definition of matrix frames is not this general idea, but the particular conditions for the accessibility relations and the intended application as a formalization of (epistemic) two-dimensional semantics.

Let me illustrate how we can represent two-dimensional intensions with models based on matrix frames using the earlier example of "Hesperus is Phosphorus" and the two scenarios and worlds used above. To give a formal representation of its two-dimensional intension, we take a matrix frame based on a two-element set, say $\{0,1\}$. Note that the set of points $W$ and the relations 
$R_{\square}, R_{@}$ and $R_{A}$ are determined by the choice of this set. We can turn this into a model $\mathfrak{M}$ by adding a valuation function $V$. Intending $p$ to stand for "Hesperus is Phosphorus", we let $V(p)=\{\langle 0,0\rangle,\langle 1,0\rangle\}$. We can then verify that $\mathfrak{M},\langle 0,0\rangle \Vdash \square p$ and $\mathfrak{M},\langle 0,0\rangle \Vdash \neg A p$.

To motivate that we have correctly captured the semantics of "actually" according to two-dimensional semantics, note that the formula $A(p \leftrightarrow @ p)$ is true in every point of every model based on a matrix frame, and that it is easy to come up with such models in which $\square(p \leftrightarrow @ p)$ is false in all points (e.g., take the matrix frame based on $\{0,1\}$ and add a valuation function $V$ with $V(p)=\{\langle 0,0\rangle,\langle 1,1\rangle\})$. This fits the claim endorsed by two-dimensionalists that indexicals can give rise to contingent a priori truths, which is already suggested in (Kaplan, 1989, pp. 538-539). See, e.g., (Chalmers, 2006b, p. 125) for an endorsement, but also Chalmers (2011a) for complications concerning the case of $p \leftrightarrow @ p$ which are not taken into account by the above semantics.

If $p$ is actually the case then $p$, and one might expect this to be reflected in the logic of actuality in the sense that it contains $@ p \rightarrow p$ as a theorem. This is not the case for the logic characterized by $\mathrm{M}$, as in all but the simplest matrix frames, the accessibility relation $R_{@}$ used to interpret the "actually" operator is not reflexive. The same issue arises in a natural formal semantics for the logic of necessity and actuality such as the definition of so-called general validity in Crossley and Humberstone (1977). Not counting @ $p \rightarrow p$ as a theorem has been defended on philosophical grounds, e.g., in Hanson (2006). One way to do so is to note that $\square(@ p \rightarrow p)$ is clearly not valid, as the sentence "Necessarily, if actually there are no talking donkeys then there are no talking donkeys" is false - assuming that there are no talking donkeys but there could be such animals. If we add the assumption that any valid formula has a valid necessitation, it follows that @ $p \rightarrow p$ is not valid. In the literature, the latter assumption is often denied, and a variant definition of validity is used that counts @ $p \rightarrow p$ but not $\square(@ p \rightarrow p)$ as valid, such as the one called real-world validity in Crossley and Humberstone (1977). This is done, e.g, in Montague (1970) and Kaplan (1989).

It is beyond the scope of this article to properly argue for one side of the dispute on whether validity implies necessity in the sense described above. Thus I will just go with the majority in the literature and claim that this is not the case, without giving an argument. Furthermore, I will adapt the definition of real-world validity to matrix frames, which gives us a formal semantics that counts $@ p \rightarrow p$ but not $\square(@ p \rightarrow p)$ as valid. Since the relation $R_{@}$ is reflexive on the diagonal points of any matrix frame, this can be done by restricting the quantification over points in the definitions of validity and consequence to points on the diagonal of the relevant matrix frame.

Although this restriction makes the logic somewhat more difficult to handle, it is technically not too unusual. In fact, it perfectly fits a variant of Kripke frames which are called frames with distinguished elements, in short FWDE, in Segerberg (1971). FWDEs are structures like Kripke frames, except that a subset of the set of points is added as a further component. The points in this set are called the distinguished points or elements. Models are obtained as in the case of frames by adding a valuation function, and the definition of the truth of a formula at a point stays the same as well. Only the definitions of validity and consequence are changed by restricting them to distinguished points. That is, for any class of FWDEs C, a formula $\varphi$ is valid on $\mathrm{C}$ if it is true at any distinguished point in any model based on any FWDE in C; and $\varphi$ is a consequence of a set of 
formulas $\Gamma$ over $\mathrm{C}$ if $\varphi$ is true at any distinguished point in any model based on any FWDE in $C$ at which all elements of $\Gamma$ are true.

Logics characterized by classes of Kripke frames are normal, which means that they contain classical propositional logic and the distributivity axioms $K_{\nabla}=\nabla(p \rightarrow q) \rightarrow(\nabla p \rightarrow \nabla q)$, and are closed under modus ponens, uniform substitution and generalization (the rule saying that if $\varphi$ is a theorem, so is any $\nabla \varphi$ ). Not all logics characterized by classes of FWDEs are normal, since they need not be closed under the rule of generalization. But they are quasinormal, which means that they contain the smallest normal modal logic $\mathbf{K}$ and are closed under the rules of modus ponens and uniform substitution.

We can now use the class of FWDEs obtained from adding the points on the diagonal as distinguished points to each matrix frame. I call these matrix FWDEs:

Definition 2. A matrix FWDE is an FWDE $\mathcal{F}=\left\langle W, R_{\square}, R_{@}, R_{A}, D\right\rangle$, such that $\left\langle W, R_{\square}, R_{@}, R_{A}\right\rangle$ is a matrix frame based on a set $S$ and $D=\{\langle x, x\rangle: x \in S\}$. Let $\mathrm{MD}$ be the class of matrix FWDEs.

I will use MD as the formal semantics of our propositional modal logic which is supposed to capture the relevant aspects of two-dimensional semantics. Note that since $@ p \rightarrow p$ is valid on MD but $\square(@ p \rightarrow p)$ is not, this logic is quasinormal but not normal, and therefore not characterized by any class of frames.

The question whether $@ p \rightarrow p$ should be counted as valid has an analog using $A$ in the present setting. If $p$ is a priori then $p$ is the case, and one might expect this to be reflected in the logic of apriority in the sense that it contains $A p \rightarrow p$ as a theorem. This is not the case for the logic characterized by $\mathrm{M}$, as in all but the simplest matrix frames, the accessibility relation $R_{A}$ used to interpret apriority is not reflexive. Since this relation is reflexive on the diagonal points of any matrix frame, $A p \rightarrow p$ is valid on $\mathrm{MD}$, although its necessitation $\square(A p \rightarrow p)$ is not valid on MD. Analogously to $@ p \rightarrow p$, one could try to use this fact to argue that therefore, $A p \rightarrow p$ should not be valid either, relying on the controversial premise that any validity has a valid necessitation. One difference between the cases of actuality and apriority is that while it is clear that $\square(@ p \rightarrow p)$ should not be valid, it might seem that $\square(A p \rightarrow p)$ should be valid. Since $\square(A p \rightarrow p)$ is neither valid on MD nor on $\mathbf{M}$, this is an issue that is independent of the present discussion concerning the correct definition of validity. It will be taken up in the discussion of the nesting problem below.

\section{Some Properties of the Logic}

In this section, I consider some philosophically interesting properties of the logic characterized by MD. The first is the fact that the philosophically implausible simplification of identifying scenarios and worlds in matrix frames and FWDEs does not affect the logic of the language considered here. As noted above, it would be more adequate if matrix frames were generalized to look like tables that contain at least as many rows as columns and determine a function that maps every row (representing a scenario) to a column (representing its possible world). More formally, we can define:

Definition 3. A rectangular frame is a frame $\mathfrak{F}=\left\langle W, R_{\square}, R_{@}, R_{A}\right\rangle$, such that $W=P \times S$ for some sets $P$ (representing possible worlds) and $S$ (representing 
scenarios), there is a surjective function $d: S \rightarrow P$ (representing the function that maps scenarios to their possible worlds), and the relations are given by the following conditions:

- $\langle x, y\rangle R_{\square}\left\langle x^{\prime}, y^{\prime}\right\rangle$ iff $y=y^{\prime}$

- $\langle x, y\rangle R_{@}\left\langle x^{\prime}, y^{\prime}\right\rangle$ iff $y=y^{\prime}$ and $x^{\prime}=d\left(y^{\prime}\right)$

- $\langle x, y\rangle R_{A}\left\langle x^{\prime}, y^{\prime}\right\rangle$ iff $x^{\prime}=d\left(y^{\prime}\right)$

$A$ rectangular FWDE $i s$ an FWDE based on a rectangular frame such that the set of distinguished elements $D=\{\langle d(x), x\rangle: x \in S\}$.

It is not hard to see that each model based on a rectangular frame can be expanded to be isomorphic to a model based on a matrix frame, while satisfying the same formulas. Since every matrix frame is a rectangular frame, it follows that the two classes of frames characterize the same logic, in both the sense of having the same validities as well as determining the same consequence relation. Both of these claims extend to the corresponding classes of FWDEs. (These observations also follow from results proven in Fritz (unpublished).)

This means that on the level of the propositional logic of necessity, actuality and apriority, the simplification of not distinguishing between scenarios and worlds made in matrix frames is not problematic. One might also wonder whether there could be worlds that are not the world of any scenario, which would correspond to dropping the requirement for $d$ to be surjective in the definition of rectangular frames, or whether the space of worlds could vary with the scenario (see (Chalmers, 2004, pp. 213-214) for motivation). As above, we can argue that the corresponding classes of FWDEs determine the same logic as MD. It should be noted that these observations do not in general extend to languages containing additional operators, e.g., propositional quantifiers or the so-called fixedly operator used in Davies and Humberstone (1980). See also (Humberstone, 2004, p. 20) and (Restall, 2012, pp. 1621-1622) for similar observations.

I now consider the validity of some philosophically relevant formulas. Given that proponents of two-dimensional semantics usually assume that apriority does not imply necessity and necessity does not imply apriority, it is not surprising that neither $A p \rightarrow \square p$ nor $\square p \rightarrow A p$ is valid on MD. While necessity does not imply apriority, one might think that $p$ being necessary implies that it is a priori that if $p$ is the case then $p$ is necessary. But according to the logic, this is not the case, since $\square p \rightarrow A(p \rightarrow \square p)$ is not valid on MD. That this fits our understanding of apriority and necessity has been argued in (Edgington, 2004, p. 11; the argument is attributed to Timothy Williamson, in conversation).

Logics of necessity often contain $\square p \rightarrow p$ and $p \rightarrow \square \diamond p$ as theorems, and these are valid on MD as well. More generally, the logic of MD is a conservative extension of the strong modal logic S5, in the sense that the formulas valid on MD that contain no modal operators besides $\square$ (i.e., neither @ nor $A$ ) are exactly the theorems of S5. The same holds for $A$. Furthermore, the analogous fact holds for the logic of necessity and actuality of Crossley and Humberstone (1977) according to real-world validity, as the logic of MD is also a conservative extension of that logic. These claims can be proven using structural connections between MD and well-known classes of structures that characterize these logics. 
We can also provide a syntactic characterization of the logic of MD. To do so, we first define a logic $\mathbf{2} \mathbf{D g}$, which can be proven to be the logic characterized by the class of matrix frames $\mathrm{M}$, as follows:

Definition 4. Let $\mathbf{2} \mathrm{Dg}$ be the normal modal logic axiomatized by the following formulas:

$$
\begin{array}{llll}
T_{\square} & \square p \rightarrow p & I 2 & @ p \rightarrow \square @ p \\
5_{\square} & \nabla p \rightarrow \square \vee p & 4_{A} & A p \rightarrow A A p \\
D_{@} & @ p \rightarrow \neg @ \neg & 5_{A} & C p \rightarrow A C p \\
D_{c} @ & \neg @ \neg p \rightarrow @ p & I 3 & A p \rightarrow @ p \\
I 1 & \square p \rightarrow @ p & I 4 & A(@ p \rightarrow p)
\end{array}
$$

The first six axioms are just the axioms of necessity and actuality as found in Davies and Humberstone (1980). So we only have to add the last four to express the logic of apriority and its interaction with necessity and actuality. Using $2 \mathbf{D g}$, we can derive a logic $\mathbf{2 D}$, which can be proven to be the logic characterized by the class of matrix FWDEs MD:

Definition 5. Let 2D be the logic such that $\varphi$ is a theorem of 2D if and only if $@ \varphi$ is a theorem of $\mathbf{2} \mathbf{D g}$.

Theorem 6. $2 \mathrm{Dg}$ is sound and strongly complete with respect to $\mathbf{M} . \mathbf{2 D}$ is sound and strongly complete with respect to MD.

This is proven in Fritz (unpublished) along with a number of further results concerning $\mathbf{2 D}$.

Instead of using MD, there are also other ways of giving a formal semantics for the propositional language considered here that can be claimed to capture the ideas of two-dimensional semantics, since in a number of respects, the informality of the expositions of two-dimensional semantics leaves room for decisions. An example would be to differentiate between contexts of utterance and scenarios, and consequently give a semantics using triples instead of pairs. This is motivated by the fact that two-dimensional semantics does not commit us to the claim that the dependence of words like "Hesperus" and "water" on the scenario relative to which they are evaluated is the same as the dependence of indexicals like "I" and "actually" on the context in which they are uttered, as noted in (Chalmers, 2006a, p. 599). Distinguishing between contexts of utterance and scenarios in the formal semantics also provides greater flexibility in defining validity and consequence. E.g., one could then define validity in a way that would not count @ $p \rightarrow p$ as valid, and in general, would give us the logic of necessity and actuality of Crossley and Humberstone (1977) according to general validity, while still counting $A p \rightarrow p$ as valid. Therefore, the present development should only be seen as one way of giving a logic based on two-dimensional semantics, although, I hope, a natural and simple one.

The formal syntax and semantics defined in the previous section are specifically tailored to the epistemic variant of two-dimensional semantics considered here. Thus they might, but need not, be applicable as formalizations of other variants of two-dimensional semantics. As an example, consider the semantics of indexicals discussed in Kaplan (1989). If we omit the relation interpreting the operator $A$ and take a point of a matrix frame to represent a world of evaluation and a world of utterance, then we get a formalization of the logic of necessity 
and "actually" which fits Kaplan's semantic account. But if we add the relation interpreting $A$ as it is defined for matrix frames, we cannot understand this as providing a semantics of "it is a priori that", or indeed any expression of the form $\ulcorner$ it is $X$ that $\ldots\urcorner$ in English. This is because such an expression would then have to shift the context of utterance in the course of evaluating a sentence, and Kaplan claims that there are no such expressions in English. See (Kaplan, 1989, pp. 510-512), where he calls them monsters.

As Kaplan notes, we are able to make claims in English that require evaluating expressions in other contexts, by mentioning rather than using the expression, and explicitly quantifying over contexts. This opens up an interpretation of $A$ that is consistent with Kaplan's ban on monsters. In particular, we can understand $A$ as standing for $\ulcorner$ in all contexts '...' is true $\urcorner$. E.g., the formula $A(@ p \rightarrow p)$ could stand for "In all contexts 'If it is actually raining then it is raining' is true". What this example demonstrates is that the present formalization, on which $A$ represents apriority and points in matrix frames represent world-scenario pairs, is specific to epistemic two-dimensional semantics. While the formal syntax and semantics are abstract enough to be applicable to other variants of two-dimensional semantics, this has to be argued for in each particular case, and some aspects of the formal apparatus might have to be re-intepreted to allow it.

\section{The Nesting Problem}

I have given a formalization of two-dimensional semantics in the form of a formal semantics for a modal logic in a propositional language with operators for necessity, actuality and apriority. This answers Dever's challenge of providing a detailed story about the interactions of primary and secondary intensions, at least for the very restricted fragment of natural language which the formal syntax used here is capable of representing.

We can also apply the formalization to the nesting problem. Its central argument can be written as follows in the formal language, where $N 1$ and $N 2$ represent the premises, and $N 3$ the conclusion:

$$
\begin{array}{ll}
\text { N1 } & A p \rightarrow \square A p \\
\text { N2 } & \square(A p \rightarrow p) \\
\text { N3 } & A p \rightarrow \square p
\end{array}
$$

While the conclusion is a consequence of the premises over MD and the first premise is valid on MD, the second premise and the conclusion are not. That is, $\{N 1, N 2\} \vDash_{\mathrm{MD}} N 3, \mathrm{MD} \Vdash N 1$, MD $\nVdash N 2$, and MD $\nVdash N 3$. To see that $N 2$ and $N 3$ are not valid on MD, consider a model in which $p$ is true throughout the diagonal, but not throughout the horizontal, e.g., the model obtained from adding a valuation function $V$ with $V(p)=\{\langle 0,0\rangle,\langle 1,1\rangle\}$ to the matrix FWDE based on $\{0,1\}$. So according to the formalization, proponents of two-dimensional semantics should answer the nesting problem by denying that all instances of the second premise are true. In particular, they should claim that if $p$ is a priori and contingent, then it is not necessarily the case that if $p$ is a priori then $p$ is the case, and thus possible that $p$ is a priori and $p$ is not the case. 
This might seem strange - it might seem more plausible to deny the validity of $N 1$ than to deny the validity of $N 2$. Of course, the mere fact that $N 2$ looks more plausible than $N 1$ at first glance is far from conclusive evidence that $N 2$ rather than $N 1$ is a theorem of the correct logic of necessity and apriority. But this fact does pose the challenge to give philosophical reasons for counting $N 1$ rather than $N 2$ as valid, and so far, we have not seen any arguments to this conclusion. So although the formalization developed above indicates a possible answer to the nesting problem, this may not be the correct answer, or the best answer two-dimensional semantics can offer. E.g., (Chalmers, 2011b, endnote 25) discusses a modification of the analysis of apriority in two-dimensional semantics which gives a different answer to the nesting problem. This is worked out in more detail in Chalmers and Rabern (unpublished).

We can thus distinguish three possibilities. Firstly, it could be that the assessment of the nesting argument suggested by $\mathbf{2 D}$ (i.e., that $N 1$ is valid, $N 2$ and $N 3$ are invalid, and $N 3$ is a consequence of $N 1$ and $N 2$ ) is correct. Secondly, it could be that this assessment is incorrect, but that there is a way of amending two-dimensional semantics or its formalization to reflect the correct assessment. And thirdly, it could be that the assessment suggested by $\mathbf{2 D}$ is incorrect, and that there is no way of amending two-dimensional semantics or its formalization to reflect the correct assessment. Only in the third case does the nesting problem offer an argument against two-dimensional semantics.

I am unsure what the correct assessment of the nesting argument is, and I am also unsure whether there is a good way of amending two-dimensional semantics or its formalization to allow for a different assessment of the nesting argument. Therefore, I will confine myself to pointing out some of the difficulties involved in assessing the nesting problem in the remainder of this section. It is clear that the issues involved in doing so are important for deciding what the nesting problem tells us about two-dimensional semantics. But I think that they are also of wider interest: $N 1$ seems like a plausible principle independently of two-dimensional semantics. The main reason for feeling compelled to give it up when confronted with the nesting problem is that $N 2$ seems even more plausible. Therefore, I think that the nesting problem should be seen as a puzzle that is interesting independently of two-dimensional semantics.

I start with a very natural but problematic argument for the validity of $N 1$, which is described in (Chalmers, 2011b, endnote 25). It goes as follows: for $p$ to be a priori is for $p$ to be a priori knowable, i.e., for it to be possible that someone has a priori knowledge that $p$. So if we write $A^{*} p$ for someone having a priori knowledge that $p$, we can write $\diamond A^{*} p$ for $A p$. Thus we can write $N 1$ as $\diamond A^{*} p \rightarrow \square \diamond A^{*} p$. Since this is a substitution instance of $5_{\square}=\diamond p \rightarrow \square \diamond p$, which is a theorem of $\mathbf{S 5}$, it is valid.

There are two problems with this argument. The first is that it assumes that the relevant modality in "a priori knowable" is the modality which we have been talking about so far, and which we formalize using $\square$ and $\diamond$. We can give an example for a reason to doubt this if we assume that one has to be in causal contact with a substance to entertain contents about it and that there are possible substances $s$ and $s^{\prime}$ that are not compossible. In this case, it is impossible to entertain and thus impossible to know a priori that everything that is $s$ is $s$ and everything that is $s^{\prime}$ is $s^{\prime}$. However, it is plausible that this conjunction is still a priori.

The second problem with the proposed argument for $N 1$ is that it not only 
requires us to assume that $p$ is a priori if and only if it is possible for someone to have a priori knowledge that $p$, but that this biconditional is necessary. If we take "it is a priori that" to be an abbreviation for "it is possible that someone has a priori knowledge that", this stronger assumption is plausible, but if we only take the former phrase to be introduced or explained using the latter phrase, the necessitated biconditional is no longer obvious. In particular, one could take the nesting argument to show that we are uncertain about how to understand claims about apriority that are embedded in modal operators. Since the relevant necessitated biconditional is exactly such a claim, we cannot use it as a premise in an argument for a solution to the nesting problem.

Another natural but problematic way of arguing for one of the premises of the nesting problem uses quantified statements. E.g., it is natural to argue for $N 2$ by noting that whatever is a priori is the case, and that this is not contingently so (we're not lucky to be in a world in which apriority implies truth). So necessarily, what is a priori is the case, and this is just $N 2$ (at least if we assume that necessarily, entities of the relevant kind have necessary being). We can give a similar argument for $N 1$, by claiming that what is a priori is not a contingent matter (e.g., we're not lucky to be in a world in which it is a priori that what is actually the case is the case), and so that what is a priori is necessarily a priori, which is just $N 1$.

Assuming that the correct response to the nesting problem is to reject one of the premises, not both of these arguments can be correct. Two-dimensional semantics has a natural way of rejecting both of the arguments, by pointing out that it is unclear what the domain of quantification is in them. In twodimensional semantics, there are several kinds of entities that play some of the roles traditionally attributed to propositions. E.g., there are primary, secondary and two-dimensional intensions, and from these, various notions of structured propositions can be derived; see Chalmers (2011b). To see why this might create problems for the above arguments, consider the argument for N1. Given the analysis of apriority of two-dimensional semantics, it is plausible that we quantify over primary intensions when we say that what is a priori is the case. If we then conclude that this is the case necessarily, we have to evaluate such a primary intension in counterfactual worlds, although primary intensions are functions on scenarios, not worlds. It is unclear how such an evaluation should be represented in two-dimensional semantics, but it is clear that it provides a way of accounting for false pre-theoretic judgements. It would be an interesting project to add propositional quantifiers to the formal language considered here and find out how they interact with necessity and apriority, but this is beyond the scope of the present paper.

One response to the problems with arguments relying on propositional quantification is to concentrate on instances of the relevant formulas. E.g., instead of asking whether there is something that is a priori but not necessarily a priori, we might consider whether there is a false instance of the sentence schema (A1) given in section 1. However, if we consider the formalized version of the nesting argument presented at the beginning of this section, we don't only have to consider the methodological question whether it is more fruitful to consider quantified versions or instances of the relevant formulas, but also the question what the correct notion of logical validity requires. E.g., according to the standard of validity for propositional modal languages proposed in (Williamson, forthcoming $\mathrm{b}$, chapter 3$)$, a formula such as $\square(A p \rightarrow p)$ is valid if and only if its 
universal closure $\forall p \square(A p \rightarrow p)$ is true on its intended interpretation. On such an account of validity, the truth or falsity of natural language sentences might not tell us everything we need to know, especially if it is assumed that the intended interpretation of a formal language need not be given by any translation into natural language.

An account of validity that counts a formula as valid if and only if all of its natural language instances are true may seem to be easier to evaluate with respect to our pre-theoretic judgement, but such an account is also not without its complications. As an example, consider the formula $(A p \wedge \neg \square p) \rightarrow \diamond(A p \wedge$ $\neg p$ ), which follows in any quasi-normal modal logic from $N 1$. If this formula is valid, then it follows that for any contingent a priori truth, the relevant instance of $\diamond(A p \wedge \neg p)$ is true. So defending the validity of $N 1$ seems to commit us to the truth of the following natural language sentence (using parentheses to resolve ambiguities):

(B) Possibly, (it is a priori that (grass is green if grass is actually green) and it is not the case that (grass is green if grass is actually green)).

It is just not obvious whether (B) is true or false. Even if we are inclined to judge it as false on pre-theoretic grounds, this doesn't mean that this judgment can't be overturned by theoretical considerations. E.g., one could argue that (B) is in fact true, and only seems false since the occurrence of "actually" within the scope of "a priori" functions differently from the occurrence outside of it.

We could also argue that it is irrelevant whether (B) is true, since despite appearances, (B) is not an instance of $\diamond(A p \wedge \neg p)$. One reason for this might be that the natural reading of $(\mathrm{B})$ is what we might call de re with respect to "actually". More precisely, we could claim that the natural reading of (B) can be paraphrased as follows:

(B') Actuality is such that possibly, (it is a priori that (grass is green if grass is green in it) and not the case that (grass is green if grass is green in it)).

If the natural reading of $(B)$ can in fact be paraphrased by $\left(\mathrm{B}^{\prime}\right)$, then we can argue that the natural reading of $(\mathrm{B})$ is not an instance of $\diamond(A p \wedge \neg p)$, as the relevant facts about scope have to be represented in the formula. This can be substantiated by noting that analogously, arguments containing anaphora can appear to provide counterexamples to classical first-order logic if the anaphora are not explicitly represented; see, e.g., (van Benthem, 1987, p. 464).

However, if this is correct, then it is inadequate to formalize "actually" using a unary sentential operator @, and therefore also to formalize two-dimensional semantics as it was done above. One might try to save the formalization by removing the @ operator from the syntax and semantics, but if indexicals like "actually" have to be represented explicitly in formulas capturing sentences in which they occur, then no sentence containing an indexical can be captured in the formalization. Since many examples of contingent a priori truths contain indexicals, this would severely limit its interest.

Both of these strategies for reconciling a pre-theoretic judgement that (B) is false with the claim that $N 1$ is valid essentially rely on indexicals in the relevant statements. But as Williamson (1986) has argued, not all contingent a priori truths involve indexicals. Williamson's example is the truth that there is at least one believer, where a believer is anyone who believes. Thus on the account 
of validity considered at the moment, accepting $N 1$ as valid seems to commit us to the truth of the following claim:

(C) Possibly, it is a priori that there is at least one believer and there is no believer.

This seems difficult to accept. Overall, we see that even if we accept a standard of validity that depends on the truth of natural language instances of formulas, the natural language data are difficult to assess, as well as the question which data are relevant.

From the preceding discussion, it might seem that formalizing the nesting problem in a propositional modal logic of necessity and apriority only makes answering it harder. In the original formulation, we only had to find out which instances of the sentence schemas (A1)-(A3) are true. Now, we also have to consider what the correct standard for validity is, and how to connect natural language sentences to formulas. While this is true, the formalization may help us to assess the truth of the original sentences by forcing us to answer certain questions, e.g., about the nature of propositions and how to quantify over them.

The difficulties with the nesting problem disappear if we interpret $A$ as standing for $\ulcorner$ in all contexts '...' is true $\urcorner$, since it then reads as follows:

(D1) If in all contexts ' $p$ ' is true, then it is necessary that in all contexts ' $p$ ' is true.

(D2) Necessarily, if in all contexts ' $p$ ' is true, then $p$.

(D3) If in all contexts ' $p$ ' is true, then it is necessary that $p$.

There is no reason for taking (D2) to be true in general, as, e.g., "Necessarily, if in all contexts 'grass is green if grass is actually green' is true, then grass is green if grass is actually green" is false. But re-interpreting $A$ is not solving the nesting problem; it is merely moving to a different and less problematic argument. We still want to know which of the premises in the original argument to reject.

A similar proposal treats "a priori" as a predicate of sentences rather than a sentential operator. It is plausible that this also avoids the nesting problem. But the claim that "a priori" is a predicate of sentences is implausible. For consider the English sentence "It is a priori that three is prime" and its German translation "Es ist a priori dass drei prim ist". These sentences seem to be about the same subject matter. But if we understand apriority as a sentential predicate, the corresponding sentences are about different things: "Three is prime' is a priori" is about "Three is prime", while "Drei ist prim' ist a priori" is about "Drei ist prim". Treating apriority as a sentential predicate also gives rise to problems of self-reference similar to the familiar problems for truth predicates. This was already observed for necessity in Montague (1963); see Halbach (2009) and Schwarz (forthcoming) for recent discussion.

Of course, one could also reject the notion of apriority outright, and thus attempt to dissolve the nesting problem. Reasons for dissatisfaction with the notion of apriority that are unconnected to the nesting problem are discussed, e.g., in Williamson (forthcoming a). For a proponent of two-dimensional semantics, this is not an appealing strategy, as much of two-dimensional semantics is based on the intelligibility of the notion. It also doesn't suffice as a strategy for dealing with the nesting problem in its full generality, as a variant of the 
nesting problem can be formulated without "a priori", using the terms "knows", "always" and "now" instead of "a priori", "necessary" and "actually".

To see this, let $K$ stand for "the agent knows", $L$ for "always" and $N$ for "now". Then just as @ $p \rightarrow p$ is valid, so is $N p \rightarrow p$. Since we formalize perfectly rational agents in epistemic logic, the modeled agent always knows every validity, so in particular $L K(N p \rightarrow p)$. Further, it is always the case that if $p$ is known by the agent then $p$ (i.e., $L(K p \rightarrow p)$ ). As a substitution instance, we obtain $L(K(N p \rightarrow p) \rightarrow(N p \rightarrow p))$, so in any quasi-normal modal logic, it follows that $L(N p \rightarrow p)$. But given the plausible assumption that if $p$ is the case then it is always now the case that $p$ (i.e., $p \rightarrow L N p$ ), it follows that if $p$ is the case then $p$ is always the case (i.e., $p \rightarrow L p$ ). This is surely not valid - although there are humans, it is not always the case that there are humans. The analog to $N 1$ is the rule that $L K \varphi$ is valid for all validities $\varphi$, and the analog to $N 2$ is the formula $L(K p \rightarrow p)$. While initially, it also looks highly implausible to reject the latter principle, closer inspection might give us reason to doubt its validity.

What this variant using the temporal expressions "always" and "now" and the epistemic expression "knows" demonstrates is that problems similar to that of the nesting problem can be formulated without relying on notions such as apriority and necessity. This emphasizes the point that the nesting problem is not specific to two-dimensional semantics, and illustrates that it cannot be solved simply by rejecting notions such as apriority or necessity as unintelligible. Rather, it indicates that the nesting problem is independent of any particular theory and arises out of the combination of epistemic and non-epistemic modalities with indexicals.

\section{Conclusion}

The central claims of epistemic two-dimensional semantics about meaning using primary and secondary intensions and their relations to necessity and apriority as well as their interactions with "actually" can be systematized using standard ways of providing a combinatorial semantics. Here, this was done using a propositional modal language and a formal semantics based on a class of Kripke frames with distinguished elements. This allows us to tell at least a partial story about how primary and secondary intensions interact. Doing so in the way presented here provides us with a logic of necessity, actuality and apriority, which gives us further insights about the relation of these modalities according to epistemic two-dimensional semantics.

The logic can also be used to formalize the nesting problem, and indicates one way of answering it, namely accepting the first and rejecting the second premise. We have seen that it is difficult to decide whether this is the right response, and I have noted that even if it is not, there might be ways of amending epistemic two-dimensional semantics or its formalization to provide a different response. It is thus not clear what the nesting problem tells us about epistemic two-dimensional semantics. Finally, we have seen that the nesting problem is puzzling independently of two-dimensional semantics, and that variants can be formulated that do not rely on the modalities of necessity and apriority. 


\section{Acknowledgements}

This paper is based on my master's thesis, written in 2011 at the ILLC, University of Amsterdam, and supervised by Paul Dekker and Yde Venema. I thank them, as well as David Chalmers, Jeremy Goodman, Emar Maier, Peter Pagin, Brian Rabern, Robert van Rooij, Wolfgang Schwarz and Timothy Williamson for discussions on various stages and predecessors of this paper. I also thank audiences in Cologne, Amsterdam, Stockholm and London as well as two anonymous reviewers for comments. I especially thank Robert Michels for many helpful discussions and detailed written comments on several drafts of this paper. This article was written while I was supported by a doctoral studentship from the Arts and Humanities Research Council.

\section{References}

David J. Chalmers. The Conscious Mind. Oxford: Oxford University Press, 1996.

David J. Chalmers. Epistemic two-dimensional semantics. Philosophical Studies, 118:153-226, 2004.

David J. Chalmers. Two-dimensional semantics. In Barry C. Smith and Ernest Lepore, editors, The Oxford Handbook of Philosophy of Language, pages 574606. Oxford: Oxford University Press, 2006a.

David J. Chalmers. The foundations of two-dimensional semantics. In Manuel García-Carpintero and Josep Macià, editors, Two-Dimensional Semantics, pages 55-140. Oxford: Oxford University Press, 2006b.

David J. Chalmers. Actuality and knowability. Analysis, 71:411-419, 2011a.

David J. Chalmers. Propositions and attitude ascriptions: A Fregean account. Noûs, 45:595-639, 2011b.

David J. Chalmers and Brian Rabern. Two-dimensional semantics and the nesting problem. Typescript, unpublished.

John N. Crossley and Lloyd Humberstone. The logic of "actually". Reports on Mathematical Logic, 8:11-29, 1977.

Martin Davies and Lloyd Humberstone. Two notions of necessity. Philosophical Studies, 38:1-30, 1980.

Josh Dever. Low-grade two-dimensionalism. Philosophical Books, 48:1-16, 2007.

Dorothy Edgington. Two kinds of possibility. Aristotelian Society Supplementary Volume, 78:1-22, 2004.

Graeme Forbes. The problem of factives for sense theories. Analysis, 71:654-662, 2011.

Peter Fritz. What is the correct logic of necessity, actuality and apriority? Typescript, unpublished. 
Kathrin Glüer and Peter Pagin. Proper names and relational modality. Linguistics and Philosophy, 29:507-535, 2006.

Kathrin Glüer and Peter Pagin. Reply to Forbes. Analysis, 72:298-303, 2012.

Volker Halbach. On the benefits of a reduction of modal predicates to modal operators. In Alexander Hieke and Hannes Leitgeb, editors, Reduction Abstraction - Analysis, pages 323-333. Heusenstamm: Ontos Verlag, 2009.

William H. Hanson. Actuality, necessity, and logical truth. Philosophical Studies, 130:437-459, 2006.

Lloyd Humberstone. Review of Multi-Dimensional Modal Logic by Maarten Marx and Yde Venema. Studia Logica, 65:278-282, 2000.

Lloyd Humberstone. Two-dimensional adventures. Philosophical Studies, 118: 17-65, 2004.

Hans Kamp. Formal properties of 'now'. Theoria, 37:227-273, 1971.

David Kaplan. Demonstratives. In Joseph Almog, John Perry, and Howard Wettstein, editors, Themes from Kaplan, pages 481-563. Oxford: Oxford University Press, 1989

Saul A. Kripke. Naming and Necessity. Cambridge, MA: Harvard University Press, 1980 [1972]. First published in Semantics of Natural Language, edited by Donald Davidson and Gilbert Harman, pages 253-355, 763-769, Dordrecht: D. Reidel, 1972.

Robert Michels. Soames's argument 1 against strong two-dimensionalism. Philosophical Studies, 161:403-420, 2012.

Richard Montague. Syntactical treatments of modality, with corollaries on reflexion principles and finite axiomatizability. In Proceedings of a Colloquium on Modal and Many-Valued Logics, volume 16 of Acta Philosophica Fennica, pages 153-167. Helsinki: Societas Philosophica Fennica, 1963.

Richard Montague. Universal grammar. Theoria, 36:373-398, 1970.

Greg Restall. A cut-free sequent system for two-dimensional modal logic, and why it matters. Annals of Pure and Applied Logic, 163:1611-1623, 2012.

Laura Schroeter. Two-dimensional semantics. In Edward N. Zalta, editor, Stanford Encyclopedia of Philosophy. Winter 2010 edition, 2010.

Wolfgang Schwarz. Variations on a Montagovian theme. Forthcoming in Synthese, available online in advance of print. doi: 10.1007/s11229-012-0173-0, forthcoming.

Krister Segerberg. An Essay in Classical Modal Logic. Number 13 in Filosofiska Studier. Uppsala: Uppsala Universitet, 1971.

Krister Segerberg. Two-dimensional modal logic. Journal of Philosophical Logic, 2:77-96, 1973. 
Scott Soames. Reference and Description: The Case Against Two-Dimensionalism. Princeton: Princeton University Press, 2005.

Robert C. Stalnaker. Assertion. In Peter Cole, editor, Syntax and Semantics, Volume 9: Pragmatics, pages 315-332. New York: Academic Press, 1978.

Johan van Benthem. Meaning: Interpretation and inference. Synthese, 73: 451-470, 1987.

Timothy Williamson. The contingent a priori: Has it anything to do with indexicals? Analysis, 46:113-117, 1986.

Timothy Williamson. How deep is the distinction between a priori and a posteriori knowledge? In Albert Casullo and Joshua C. Thurow, editors, The A Priori in Philosophy. Oxford: Oxford University Press, forthcoming a.

Timothy Williamson. Modal Logic as Metaphysics. Oxford: Oxford University Press, forthcoming b. 